\title{
ARTICLE
}

\section{Computational prediction of protein subdomain stability in $M Y B P C 3$ enables clinical risk stratification in hypertrophic cardiomyopathy and enhances variant interpretation}

Andrea D. Thompson ${ }^{1 凶}{ }^{凶}$, Adam S. Helms ${ }^{1}$, Anamika Kannan ${ }^{1}$, Jaime Yob ${ }^{2}$, Neal K. Lakdawala ${ }^{3}$, Samuel G. Wittekind ${ }^{4}$, Alexandre C. Pereira ${ }^{5}$, Daniel L. Jacoby ${ }^{6}$, Steven D. Colan ${ }^{7}$, Euan A. Ashley ${ }^{8}$, Sara Saberi ${ }^{1}$, James S. Ware ${ }^{9}$, Jodie Ingles ${ }^{10}$, Christopher Semsarian ${ }^{11}$, Michelle Michels ${ }^{12}$, Francesco Mazzarotto ${ }^{9,13,14}$, lacopo Olivotto ${ }^{13,14}$, Carolyn Y. Ho ${ }^{3}$ and Sharlene M. Day ${ }^{2 凶}$

PURPOSE: Variants in MYBPC3 causing loss of function are the most common cause of hypertrophic cardiomyopathy (HCM). However, a substantial number of patients carry missense variants of uncertain significance (VUS) in MYBPC3. We hypothesize that a structural-based algorithm, STRUM, which estimates the effect of missense variants on protein folding, will identify a subgroup of HCM patients with a MYBPC3 VUS associated with increased clinical risk.

METHODS: Among 7,963 patients in the multicenter Sarcomeric Human Cardiomyopathy Registry (SHaRe), 120 unique missense VUS in MYBPC3 were identified. Variants were evaluated for their effect on subdomain folding and a stratified time-to-event analysis for an overall composite endpoint (first occurrence of ventricular arrhythmia, heart failure, all-cause mortality, atrial fibrillation, and stroke) was performed for patients with HCM and a MYBPC3 missense VUS.

RESULTS: We demonstrated that patients carrying a MYBPC 3 VUS predicted to cause subdomain misfolding (STRUM,$+ \triangle \triangle G \leq-1.2$ $\mathrm{kcal} / \mathrm{mol}$ ) exhibited a higher rate of adverse events compared with those with a STRUM- VUS (hazard ratio $=2.29, P=0.0282$ ). In silico saturation mutagenesis of MYBPC3 identified 4,943/23,427 (21\%) missense variants that were predicted to cause subdomain misfolding.

CONCLUSION: STRUM identifies patients with HCM and a MYBPC3 VUS who may be at higher clinical risk and provides supportive evidence for pathogenicity.

Genetics in Medicine (2021) 23:1281-1287; https://doi.org/10.1038/s41436-021-01134-9

\section{INTRODUCTION}

Genetic variant interpretation is an ongoing challenge in clinical medicine, particularly when the gene of interest lacks robust functional assays. ${ }^{1,2}$ A variety of computational algorithms have been developed to predict variant pathogenicity, but their sensitivity and specificity are often poor, particularly when applied broadly across different diseases and different genes. ${ }^{1,3}$ Loss-offunction (LoF) pathogenic variants are common, ${ }^{1,4,5}$ resulting from either frameshift or nonsense variants creating a premature stop codon, splice errors, disruption of enzymatic activity, alteration of protein-protein interactions, or protein misfolding. ${ }^{1,6,7}$ Recognizing a common mechanism by which variants in a particular gene lead to LoF can inform the development of gene-specific computational algorithms to more accurately predict pathogenicity among variants that cannot be confidently classified based on clinical and family data alone., ${ }^{6,7}$

Herein we focus on MYBPC3 (encoding the protein, cardiac myosin binding protein $\mathrm{C}$, or MyBP-C). Pathogenic variants in $M Y B P C 3$ account for $\sim 50 \%$ of patients with sarcomeric hypertrophic cardiomyopathy $(\mathrm{HCM}),{ }^{8,9}$ and are inherited in an autosomal dominant fashion (OMIM 115197). Patients with HCM can experience a variety of adverse clinical outcomes, including outflow tract obstruction, arrhythmias, heart failure, and sudden cardiac death. $^{8}$ Genetic variants in MYBPC3 consist of both truncating and nontruncating types. Rarely found in healthy populations, truncating $M Y B P C 3$ variants result in a premature stop codon and cause HCM through complete LoF and haploinsufficiency at the transcript and protein level. ${ }^{10-13}$ Thus, interpretation of these truncating variants as pathogenic is straightforward. ${ }^{14}$

However, the interpretation of missense variants within MYBPC3 presents a major challenge. Single amino acid substitutions (missense variants) are found commonly in healthy populations. Further, since missense variants do not disrupt the reading frame, protein function may be tolerant to these minor sequence changes. Thus, many missense variants lack sufficient evidence to be classified as either pathogenic or benign and are classified as variants of uncertain significance (VUS). ${ }^{14,15}$ While identifying pathogenic variants allows for predictive genetic testing in at-risk relatives, ${ }^{16}$ a VUS is not clinically actionable and may lead to misinterpretation by clinicians and patients. ${ }^{17}$

\footnotetext{
${ }^{1}$ Cardiovascular Medicine, University of Michigan, Ann Arbor, MI, USA. ${ }^{2}$ Cardiovascular Medicine, University of Pennsylvania, Philadelphia, PA, USA. ${ }^{3}$ Cardiovascular Medicine, Brigham and Women's Hospital, Harvard Medical School, Boston, MA, USA. ${ }^{4}$ Cincinnati Children's Hospital Medical Center, Heart Institute, Cincinnati, OH, USA. ${ }^{5} \mathrm{Heart}$ Institute (InCor), University of Sao Paolo Medical School, Sao Paulo, Brazil. ${ }^{6}$ Cardiovascular Medicine, Yale University, New Haven, CT, USA. ${ }^{7}$ Department of Cardiology, Boston Children's Hospital, Boston, MA, USA. ${ }^{8}$ Center for Inherited Heart Disease, Stanford University, Stanford, CA, USA. ${ }^{9}$ National Heart \& Lung Institute \& Royal Brompton Cardiovascular Research Centre, Imperial College London, London, United Kingdom. ${ }^{10}$ Cardio Genomics Program at Centenary Institute, The University of Sydney, Sydney, Australia. ${ }^{11}$ Agnes Ginges Centre for Molecular Cardiology at Centenary Institute, The University of Sydney, Sydney, Australia. ${ }^{12}$ Department of Cardiology, Thoraxcenter, Erasmus MC Rotterdam, The Netherlands. ${ }^{13}$ Cardiomyopathy Unit, Careggi University Hospital, Florence, Italy. ${ }^{14}$ Department of Clinical and Experimental Medicine, University of Florence, Florence, Italy.

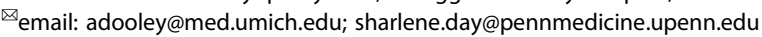


Identification of a pathogenic sarcomere genetic variant for HCM also has important prognostic implications. Patients with $\mathrm{HCM}$ and a pathogenic sarcomere variant (sarcomeric HCM) have a higher risk of adverse clinical outcomes compared with those without a sarcomere gene variant (nonsarcomeric HCM). ${ }^{8,18}$ Patients carrying a sarcomere gene VUS, on average, exhibit an intermediate risk of adverse events, ${ }^{8}$ most likely because VUS represent a mixed pool of pathogenic and benign variants that cannot be parsed on the basis of clinical and genetic data alone.

Because LoF is an established mechanism for pathogenic variants in $M Y B P C 3$, we hypothesized that applying a computational approach, called STRUM, ${ }^{19-21}$ that incorporates both sequencebased and structure-based algorithms to missense MYBPC3 VUS will identify those variants that result in protein subdomain misfolding (STRUM+), thereby supporting pathogenicity and improving variant interpretation. We further predict that this approach will identify a subpopulation of patients with HCM and a STRUM+ MYBPC3 missense VUS who are at risk for adverse clinical outcomes, at a frequency similar to patients with HCM carrying known pathogenic variants.

\section{MATERIALS AND METHODS}

Sarcomeric Human Cardiomyopathy Registry (SHaRe) data extraction and MYBPC3 variant classification

The generation of the centralized SHaRe database has been previously described. ${ }^{8}$ Data were exported from quarter 1 of 2019. Inclusion criteria included a site-designated diagnosis of HCM using standard diagnostic criteria. ${ }^{8}$ SHaRe nontruncating MYBPC3 missense variants (Tables S1, S2) were classified as previously reported ${ }^{14}$ in accordance with American College of Medical Genetics and Genomics (ACMG) and Association for Molecular Pathology (AMP) joint guidelines, leveraging available clinical and experimental data. 3,8,9,14,22,23 Known splice variants are classified as truncating. Since variants in $M Y B P C 3$ present in gnomAD with allele frequencies of $>4 \mathrm{E}-05$ and absent in $\mathrm{SHaRe}$ are unlikely to be independently pathogenic for $\mathrm{HCM}$, these variants were included in our list of benign MYBPC3 variants. ${ }^{14}$ More details regarding variant interpretation are provided within the Supplemental materials.

It has previously been shown that patients carrying pathogenic nontruncating variants exhibit similar clinical outcomes to those carrying truncating MYBPC3 variants. ${ }^{14}$ Thus, a reference population including previously adjudicated truncating and nontruncating MYBPC3 pathogenic/ likely pathogenic (pathogenic) variants (MYBPC3-path-all) was used. A second reference population included patients with HCM who underwent genetic testing and were negative for sarcomere variants Sarc- ${ }^{8}$

\section{Computational structural and protein folding stability predictive} modeling

MyBP-C is made up of immunoglobulin and fibronectin subdomains (C0-C10) (NM_000256.3, NP_000247.2). For MYBPC3 missense variants we utilized STRUM to calculate the effect of the missense variant on the Gibbs free energy of local subdomain folding $(\Delta \Delta \mathrm{G})^{19}$ (Table S3). A negative $\Delta \Delta \mathrm{G}$ value indicates the degree of reduced folding energy $(\mathrm{kcal} / \mathrm{mol})$ relative to the wild-type subdomain, or folding destabilization. ${ }^{19}$ Previous experimental validation of this algorithm compared STRUM predictions to 3,421 experimentally tested variants from 150 proteins and demonstrated a Pearson's correlation coefficient of 0.79 and root mean square error of prediction of $1.2 \mathrm{kcal} / \mathrm{mol} .{ }^{19}$ Thus, a value of $\Delta \Delta \mathrm{G} \leq-1.2 \mathrm{kcal} / \mathrm{mol}$ was defined as the cutoff for destabilizing (deleterious) variants. Further details regarding STRUM analysis and structural models are provided within the Supplemental Materials (Figure S1-S3, Table S3).

Computational sequence-based variant analysis (PolyPhen-2, SIFT, CardioBoost)

We compared the STRUM prediction for MYBPC3 missense variants with a sequence-based algorithm embedded in STRUM (SIFT). ${ }^{24,25}$ We also analyzed these variants with PolyPhen-2 (HumVar database), another sequence based algorithm. ${ }^{26}$ Finally, we compared our result with those obtained using CardioBoost, which is a disease specific machine learning classifier to predict pathogenicity of rare missense variants in genes associated with cardiomyopathies and arrhythmias. ${ }^{6}$ CardioBoost relies on minor allele frequency, whereas STRUM does not.

\section{Clinical outcomes analysis}

Only patients with HCM carrying a single MYBPC3 missense VUS were included in clinical outcomes analyses to avoid confounding from cases with multiple gene variants. ${ }^{27}$ Comparisons using time-to-event analysis were made between variants predicted to be deleterious (STRUM,$+ \Delta \Delta \mathrm{G} \leq$ $-1.2 \mathrm{kcal} / \mathrm{mol}$ ) and those predicted to be nondeleterious. The primary outcome was an overall composite previously defined as the first occurrence of any component of the ventricular arrhythmia composite, heart failure composite (without inclusions of LV ejection fraction), allcause mortality, atrial fibrillation (AF), or stroke. ${ }^{8}$ Results were compared with reference populations $M Y B P C 3$-path-all and Sarc-. A secondary analysis of a heart failure composite, ventricular arrhythmia composite, and atrial fibrillation was also performed. Finally, a secondary analysis using alternative computational algorithms (SIFT, PolyPhen-2, CardioBoost) was performed. Composite outcomes are defined in more detail in the Supplemental materials.

\section{Statistical analysis}

Data presented as mean \pm standard deviation were analyzed by $t$-test for two groups or analysis of variance (ANOVA) for $>2$ groups with Tukey's post hoc test for multiple comparisons. Data presented as frequency were analyzed by a chi-square test. Odds ratio (with $95 \%$ confidence interval $[\mathrm{CI}])$, specificity, and sensitivity were calculated to evaluate the association between computational prediction algorithms and known pathogenic/ likely pathogenic (pathogenic) or benign/likely benign (benign) variants (further details provided in supplemental materials). Primary and secondary clinical outcomes were analyzed by the Kaplan-Meier method from time of birth. Analysis from time of birth is appropriate given that the genetic variant is present from birth and variability in time to, and reason for, clinical presentation could confound the results if time from diagnosis were used. Patients who did not have the outcome of interest were censored at the time of their last recorded follow-up in SHaRe. Comparison between curves was performed using Log-rank Mantel-Cox test with $p$ values of $<0.05$ considered statistically significant. Median event free survival and hazard ratio (Mantel-Haenszel) are also reported. Statistical analyses were performed using GraphPad Prism software (San Diego, CA).

\section{RESULTS}

Patients with HCM and a MYBPC3 missense VUS predicted to disrupt subdomain folding (STRUM+) exhibit a higher incidence of adverse clinical outcomes

We began by evaluating all MYBPC3 missense VUS within SHaRe using STRUM. MYBPC3 VUS exhibited a mean $\triangle \triangle G$ of $-0.73 \pm$ $1.06 \mathrm{kcal} / \mathrm{mol}$ (Figure S4). Of 120 unique MYBPC3 missense VUS, 34 (28\%) were predicted to cause subdomain misfolding with $\Delta \Delta G$ values $\leq-1.2 \mathrm{kcal} / \mathrm{mol}$ (deleterious) (Table S2). Next, we evaluated clinical characteristics and outcomes in patients with HCM and a single missense $M Y B P C 3$ VUS predicted to disrupt subdomain folding $(S T R U M+)$ compared with patients carrying a VUS not predicted to disrupt subdomain folding (STRUM-). For this analysis, we included only patients who carried a single VUS within $M Y B P C 3$, and excluded patients who carried a second pathogenic variant or variant of uncertain significance $(N=105)$. Patients with a STRUM+ versus STRUM- MYBPC3 VUS exhibited similar clinical characteristics including body mass index (BMI), gender, ancestry, age at diagnosis, wall thickness, ejection fraction, and left ventricular outflow tract obstruction (Table 1). We observed that patients carrying a STRUM+ VUS experienced higher rates of adverse events compared with patients carrying a STRUM- VUS (Fig. 1, hazard ratio 2.3, $p=0.03$ ). Furthermore, patients carrying a STRUM + VUS exhibited a similar rate of adverse clinical events compared with patients carrying a pathogenic variant (MYBPC3Path-all). Conversely, patients carrying STRUM- VUS exhibited a lower frequency of outcomes, similar to Sarc- patients (Fig. 2). There were no statistically significant differences between groups 
Table 1. Demographics of patients with HCM and single MYBPC3 VUS.

\begin{tabular}{|c|c|c|c|}
\hline & STRUM $(+) n=39(37 \%)$ & $\operatorname{STRUM}(-) n=66(63 \%)$ & $p$ value STRUM (+) vs. STRUM (-) \\
\hline \multicolumn{4}{|l|}{ Baseline characteristics } \\
\hline Female, $n(\%)$ & $10(26 \%)$ & $14(21 \%)$ & 0.6308 \\
\hline Age at diagnosis, mean (STD), year & $40.31(17.13)$ & $41.34(21.17)$ & 0.9930 \\
\hline Follow-up time, mean (STD), year & $9.82(10.00)$ & $12.18(11.83)$ & 0.7575 \\
\hline Maximum BMl, mean (STD) kg/m² & $28.87(4.10)$ & $27.96(6.03)$ & 0.9390 \\
\hline $\mathrm{lb} / \mathrm{ft}^{2}$ & $685.09(97.29)$ & $663.49(143.09)$ & \\
\hline Race, $n \%$ & & & 0.5107 \\
\hline White & 35 (90\%) & $60(91 \%)$ & \\
\hline Black & $2(5 \%)$ & $1(2 \%)$ & \\
\hline Other/not reported & $2(5 \%)$ & $5(8 \%)$ & \\
\hline Proband, $n$ (\%) & $35(90 \%)$ & $64(97 \%)$ & 0.1232 \\
\hline Family history HCM, $n$ (\%) & $13(33 \%)$ & $19(29 \%)$ & 0.6249 \\
\hline Family history SCD $n(\%)$ & $8(21 \%)$ & 9 (14\%) & 0.3553 \\
\hline \multicolumn{4}{|l|}{ Echocardiogram data } \\
\hline Maximal LVWT, mean (STD), mm & $22.73(6.97)$ & $20.83(7.29)$ & 0.4287 \\
\hline Minimum LVEF, mean (STD), \% & $59.88(8.32)$ & $60.12(11.34)$ & 0.9996 \\
\hline LVOT peak gradient $\geq 30 \mathrm{mmHg} ; n(\%)$ & $9(23 \%)$ & $21(32 \%)$ & 0.3380 \\
\hline
\end{tabular}

BMI body mass index, HCM hypertrophic cardiomyopathy, LVEF left ventricular ejection fraction, LVWT left ventricular wall thickness, LVOT left ventricular outflow tract, SCD sudden cardiac death, VUS variant of uncertain significance.

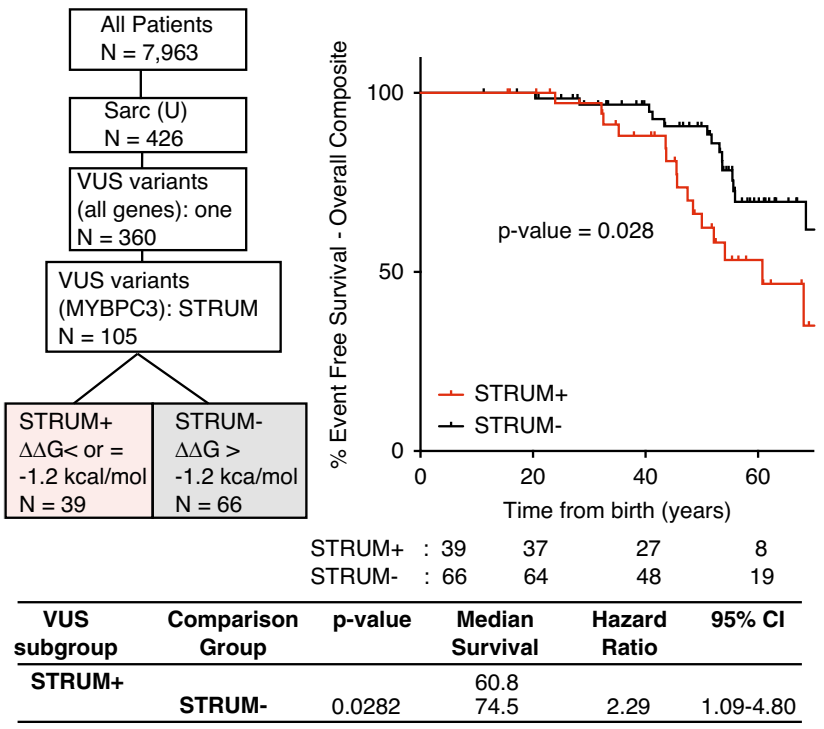

Fig. 1 Patients with a MYBPC3 VUS identified as deleterious by STRUM (STRUM + ) are associated with an increased risk for adverse hypertrophic cardiomyopathy (HCM)-related outcomes. Selection within Sarcomeric Human Cardiomyopathy Registry ( $\mathrm{SHaRe}$ ) of patients with $\mathrm{HCM}$ carrying a single MYBPC3 missense variant of uncertain significance (VUS) is shown on the left. One hundred five patients carry a single MYBPC3 missense VUS, covering 77 distinct MYBPC3 VUS. Kaplan-Meier curves, median event free survival (years), and hazard ratio with corresponding $95 \%$ confidence interval $(\mathrm{Cl})$ reveal that patients carrying a STRUM+ MYBPC3 VUS (red) exhibited a higher rate of adverse HCM-related outcomes (overall composite) compared with patients carrying a STRUMvariant (black). for the individual component outcomes, including ventricular arrhythmias, heart failure, or atrial fibrillation (Figure S5).

STRUM exhibits improved specificity over established sequencebased prediction algorithms and improved sensitivity when combined with CardioBoost

To determine the sensitivity and specificity of STRUM to differentiate pathogenic from benign variants within MYBPC3 we performed STRUM analysis on all known pathogenic missense variants within ShaRe $(n=19)$ and known missense benign variants within SHaRe and gnomAD ( $n=110$, Table S1, Fig. 3a). These variants were present in 412 patients with $\mathrm{HCM}$ within the SHaRe registry. MYBPC3 benign variants exhibited a mean $\triangle \Delta G$ of $-0.31 \pm 0.60 \mathrm{kcal} / \mathrm{mol}$, which was significantly higher than MYBPC3 VUS ( $\triangle \triangle \mathrm{G}$ of $-0.73 \pm$ $1.06 \mathrm{kcal} / \mathrm{mol}, \quad p=0.005$ ) (Figure S4) and MYBPC3 pathogenic variants (mean $\Delta \Delta \mathrm{G}$ of $-1.00 \pm 1.08 \mathrm{kcal} / \mathrm{mol}, p=0.016$ ) (Fig. 3a). We found that variants predicted to be deleterious by STRUM were more likely to be pathogenic variants (odds ratio [OR] $5.9,95 \% \mathrm{Cl}$ 1.8-19.6) (Fig. 3c). Only nine additional unique nontruncating MYBPC3 variants were designated as pathogenic and/or likely pathogenic within ClinVar. However, all of these variants had a single submission and a review status of $0-1 / 4$ criteria provided. By modern standards, these variants would be reclassified as VUS and were therefore not included in our analysis.

Algorithms that were purely sequence-based achieved greater sensitivity but performed inferiorly to STRUM in regard to specificity. STRUM exhibited a $93 \%$ specificity for benign variants and PolyPhen-2 and SIFT exhibited a specificity of $62 \%$ (OR 4.5, 95\% 1.5-13.5) and 54\% (OR 1.3, 95\% Cl 0.5-3.4) respectively (Fig. 3c, Figure S6). Additionally, variant interpretation by SIFT or PolyPhen-2 did not stratify patients carrying a MYBPC3 VUS for clinical adverse outcomes (Figure S6). 

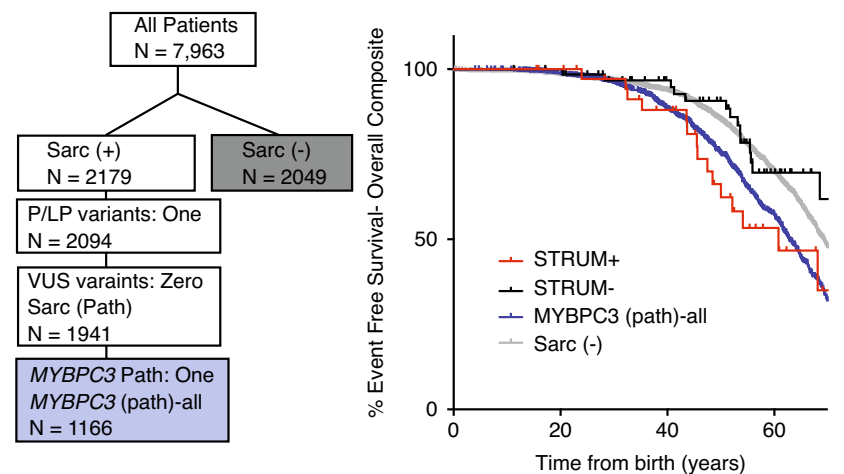

\begin{tabular}{|c|c|c|c|c|}
\hline STRUM+ & 39 & 37 & 27 & 8 \\
\hline STRUM- & $: 66$ & 64 & 48 & 19 \\
\hline MYBPC3 (Path)-all & $: 1166$ & 1078 & 768 & 267 \\
\hline Sarc (-) & : 2049 & 1913 & 1646 & 863 \\
\hline
\end{tabular}

\begin{tabular}{lccccc}
\hline $\begin{array}{l}\text { VUS } \\
\text { Subgroup }\end{array}$ & $\begin{array}{c}\text { Comparison } \\
\text { Group }\end{array}$ & p-value & $\begin{array}{c}\text { Median } \\
\text { Survival }\end{array}$ & $\begin{array}{c}\text { Hazard } \\
\text { Ratio }\end{array}$ & $95 \% \mathrm{Cl}$ \\
\hline STRUM+ & & 60.8 & & \\
& MYBPC3-Path-all & 0.5036 & 62.8 & 1.22 & $0.71-2.10$ \\
& Sarc- & 0.0136 & 69.3 & 2.32 & $1.19-4.51$ \\
\hline STRUM- & & 74.5 & & \\
& MYBPC3-Path-all & 0.0151 & 62.8 & 0.64 & $0.45-0.92$ \\
& Sarc- & 0.4996 & 69.3 & 0.86 & $0.57-1.30$ \\
\hline
\end{tabular}

Fig. 2 Patients with a MYBPC3 variant of uncertain significance (VUS) identified as deleterious by STRUM (STRUM + ) exhibit clinical outcomes similar to patients with a MYBPC3 pathogenic variants. Selection within Sarcomeric Human Cardiomyopathy Registry (SHaRe) of patients with hypertrophic cardiomyopathy (HCM) and a single MYBPC3 pathogenic variant (MYBPC3 -Path-all) and patients with HCM without a sarcomere gene variant after clinical genotype analysis (Sarc-) is shown on the left. Kaplan-Meier curves, median event free survival (median survival), and hazard ratio with corresponding $95 \%$ confidence interval $(\mathrm{Cl})$ reveal patients carrying a STRUM + MYBPC3 VUS (red) exhibited overall composite outcomes similar to MYBPC3-Path-all patients (blue, $p$ value 0.5036 ). Whereas, patients carrying a STRUM- variant (black) exhibited a lower rate of adverse HCM-related outcomes (overall composite) similar to Sarc- patients (gray).

In comparison, CardioBoost demonstrated a specificity of $98 \%$ (OR 42.3, Cl 8.0-223.6) (Fig. 3, Table S1). For pathogenic variants, CardioBoost demonstrated a sensitivity of $47 \%$. Interestingly, there was limited overlap among known pathogenic variants predicted to be deleterious by STRUM and those predicted to be deleterious by CardioBoost, making the two algorithms complementary (Table S1). Combining these algorithms to classify any variant predicted to be deleterious by CardioBoost or STRUM as pathogenic maintained a high specificity of $93 \%$ and improved sensitivity to $63 \%$ (Fig. 3c).

When examining patients with HCM and a MYBPC3 missense VUS, STRUM identified a larger number of MYBPC3 VUS as deleterious. Only 16 of 39 (41\%) patients with a STRUM+ MYBPC3 VUS were also identified as CardioBoost + . Just three additional patients were uniquely identified as CardioBoost+ (Table S2). While there is a trend toward a higher rate of adverse clinical events in patients with HCM and a CardioBoost+ MYBPC3 VUS, this difference was not statistically significant (Fig. 3d).

STRUM predictions within pathogenic variants are consistent with experimental modeling

Prior experimental characterization of $M Y B P C 3$ pathogenic missense variants within the C10 domain, Leu1238Pro and Asn1257Lys, demonstrated that these variants failed to localize a STRUM

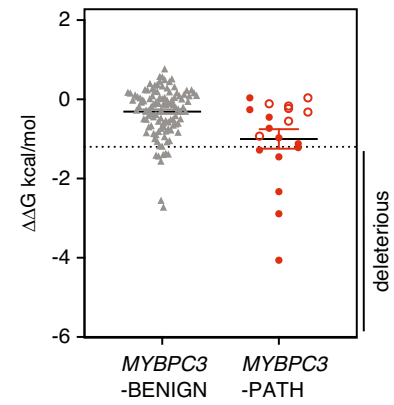

b CardioBoost

C

Comparison of algorithms

-identifying known MYBPC3 benign and pathogenic missense variants

\begin{tabular}{|c|c|c|c|c|}
\hline & Odds Ratio & $95 \% \mathrm{Cl}$ & Spec. & Sens. \\
\hline STRUM & 5.9 & $1.8-19.6$ & $93 \%$ & $32 \%$ \\
\hline SIFT & 1.3 & $0.5-3.4$ & $54 \%$ & $53 \%$ \\
\hline PolyPhen-2 & 4.5 & $1.5-13.5$ & $62 \%$ & $74 \%$ \\
\hline CardioBoos & st 42.3 & $8.0-223.6$ & $98 \%$ & $47 \%$ \\
\hline $\begin{array}{l}\text { STRUM or } \\
\text { CardioBoos }\end{array}$ & st 21.9 & $6.7-71.0$ & $93 \%$ & $63 \%$ \\
\hline
\end{tabular}

d

Overall Composite-CardioBoost

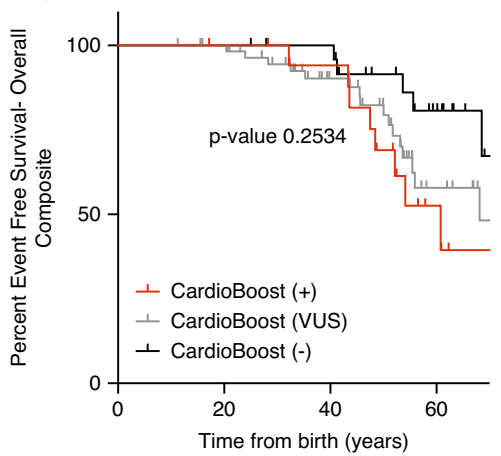

CardioBoost (+) : 19

CardioBoost (-) : 26

$\begin{array}{ccc}18 & 15 & 4 \\ 57 & 36 & 11 \\ 26 & 24 & 12\end{array}$

Fig. 3 STRUM is complementary to CardioBoost. Results of computational analysis for each unique MYBPC3-Benign (gray triangles, $n=110$ ) and MYBPC3-Path (red circles, $n=19$ ) variant. (a) STRUM. (b) CardioBoost. Mean and SEM for each group depicted. The cutoff for deleterious variants for STRUM was $\Delta \Delta \mathrm{G} \leq-1.2 \mathrm{kcal} /$ mol. The cutoff for deleterious variants for CardioBoost (CardioBoost + ) was a probability score $>0.90$; this is graphed as 1CardioBoostScore $<0.10$. C3 pathogenic variants are depicted in open circles in (a) and (b). (c) Statistical analysis of computational method utilized here in STRUM (Fig. 3), CardioBoost (Fig. 3), SIFT (Figure S6), and PolyPhen-2 (Figure S6) is shown including odds ratio, $95 \%$ confidence interval (Cl), sensitivity, and specificity. (d) Using the same patient selection criteria in the Sarcomeric Human Cardiomyopathy Registry (SHaRe) detailed in Fig. 1, patients with hypertrophic cardiomyopathy $(\mathrm{HCM})$ and a MYBPC 3 missense variants of uncertain significance (VUS) were analyzed by CardioBoost. CardioBoost $(+)$ was a probability score $>0.90$, CardioBoost VUS $\geq 0.10$ and $\leq 0.90$, and CardioBoost $(-)<0.10$. Of the 105 patients analyzed by STRUM 19 were CardioBoost $(+)$. Kaplan-Meier curves reveal that patients carrying a CardioBoost (+) MYBPC3 VUS (red) exhibited higher rates of adverse HCM-related outcomes (overall composite) than patients carrying a CardioBoost (-) MYBPC3 VUS (black); however, the null hypothesis could not be excluded, $p$ value 0.0945 . This remains true when comparing patients carrying a MYBPC 3 VUS that is CardioBoost (+) (red), CardioBoost (VUS) (gray), and CardioBoost (-) (black) ( $p$ value 0.2534$)$ 
a

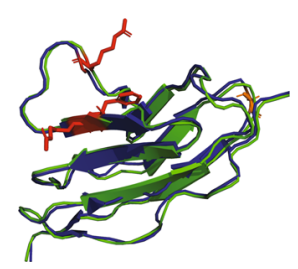

b

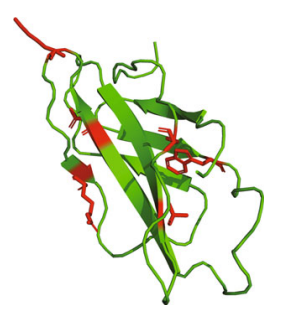

c

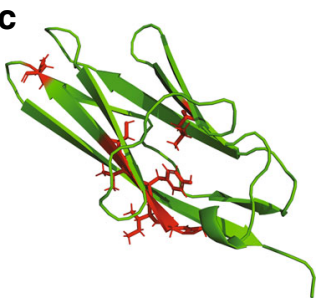

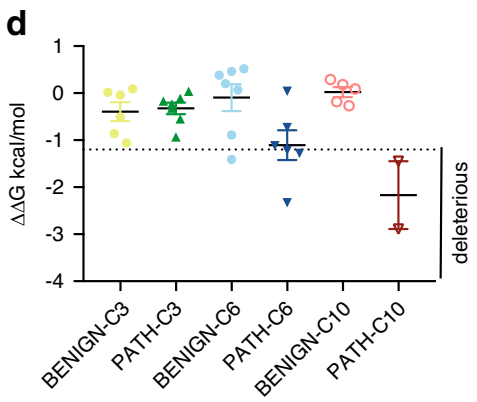

Fig. 4 Structural analysis of pathogenic missense MYBPC3 variants. MyBP-C (the protein encoded by MYBPC3) domains C3, $\mathrm{C} 6$, and $\mathrm{C} 10$ were structurally modeled using I-TASSER ${ }^{33-35}$ (PyMOL, cartoon, green). Wild-type residues that are affected by missense pathogenic variants are depicted in red (PyMOL, sticks). (a) For the C3 domain, the I-TASSER model is aligned with an available NMR structure (2mq0.pdb, ${ }^{28}$ blue, PyMOL cartoon). Pathogenic variants within C3 largely cluster in a surface-exposed region. (b) C6 domain and (c) C10 domain pathogenic variants do not cluster within a specific region of the domain. (d) Results of STRUM ${ }^{19}$ analysis for MYBPC3 pathogenic and benign variants within $\mathrm{C} 3, \mathrm{C} 6$, and $\mathrm{C} 10$ are shown, with mean and SEM for each group depicted. Graph is labeled to indicate variants predicted to be deleterious.

to the sarcomere and were rapidly degraded within primary cardiomyocytes. $^{14}$ Consistent with these experimental findings, pathogenic C10 domain variants are uniformly predicted to destabilize protein folding ( $\Delta \Delta \mathrm{G}$ of -2.89 and $-1.45 \mathrm{kcal} / \mathrm{mol}$ respectively) (Fig. 4).

Conversely, of the pathogenic MYBPC3 variants not predicted to be deleterious by STRUM (Fig. 3), a large number were localized within the $\mathrm{C} 3$ domain (Fig. 3a, open circles; 7/13) and exhibited a mean $\Delta \Delta \mathrm{G}-0.32 \mathrm{kcal} / \mathrm{mol}$, (range -0.93 to 0.04 ). A large number of known pathogenic variants cluster within the C3 domain near a surface-exposed flexible linker (Fig. 4). ${ }^{15}$ Thus, these variants would be predicted to alter electrostatic protein-protein interactions but would not be expected to disrupt subdomain folding. This result is consistent with prior experimental and structural characterization data of these C3 pathogenic variants. Arg495Gln, Arg502Trp, and Phe503Leu incorporate normally into the sarcomere and have protein half lives that are indistinguishable from wild-type MyBP-C within primary cardiomyocytes. ${ }^{14}$ Further, the NMR structure of the MYBPC3 Arg502Trp C3 domain reveals preserved subdomain folding. ${ }^{28}$

While $\mathrm{C} 3$ and $\mathrm{C} 10$ pathogenic variants have a narrow range of $\Delta \Delta \mathrm{G}$ values, $\Delta \Delta \mathrm{G}$ predictions for $\mathrm{C6}$ pathogenic variants vary from -2.33 to 0.04 (mean $\Delta \Delta G-1.11$ ). We previously examined two C6 domain variants, Arg810His and Trp792Arg, and found that they incorporate normally into the sarcomere and exhibit normal protein half lives in primary cardiomyocytes. ${ }^{14}$ However, both of these variants were predicted to destabilize subdomain folding by STRUM, exhibiting values near the cutoff: Arg810His $(\Delta \Delta \mathrm{G}-1.22 \mathrm{kcal} / \mathrm{mol})$, Trp792Arg $(\Delta \Delta \mathrm{G}-1.28 \mathrm{kcal} / \mathrm{mol})$. They are also predicted to be pathogenic by CardioBoost (Table S2). These observations suggest that a subset of pathogenic variants mildly disrupt subdomain folding without causing complete destabilization of MyBP-C.

Subdomain destabilization in these cases could interfere with protein-protein interactions or MyBP-C conformational dynamics.

In silico saturation mutagenesis of MYBPC3 identified 4,943 missense variants predicted to cause subdomain misfolding Only a subset of amino acid substitutions has been observed in patients with HCM and are cataloged in publicly available databases, such as ClinVar. However, previously unreported variants frequently arise in probands with HCM who undergo clinical genetic testing. ${ }^{29}$ Thus, we performed STRUM on all possible $M Y B P C 3$ single amino acid substitutions (in silico mutagenesis) to develop a compendium of STRUM+ variants that may be useful for the research and clinical community. We found that 4,943 of 24,665 (20\%) amino acid substitutions were predicted to disrupt subdomain folding (Figure S6, Tables S4, S5).

\section{DISCUSSION}

Clinical risk stratification has been a cornerstone of clinical HCM management. It is well-established that patients with sarcomeric HCM have a higher rate of adverse clinical outcomes compared with nonsarcomeric $\mathrm{HCM}$, enabling the incorporation of genetic data into clinical risk stratification in $\mathrm{HCM}^{8,18}$ Yet, refinement of clinical risk for patients with a VUS remains an ongoing challenge for clinicians. ${ }^{1,5}$ We have identified a subpopulation of patients with a MYBPC3 missense VUS that are predicted to disrupt subdomain protein folding (STRUM+) who exhibit clinical outcomes indistinguishable from patients with a pathogenic $M Y B P C 3$ variant. Conversely, patients carrying a MYBPC3 VUS not predicted to affect subdomain folding (STRUM-), exhibit a lower prevalence of adverse clinical outcomes similar to patients with nonsarcomeric HCM. Although the methodology of parsing these variants is different for MYBPC3 because of differing underlying mechanisms, these findings are analogous to a recent study in $\mathrm{MYH7}$ in which patients with HCM carrying VUS that were located within the interacting heads motif had a higher rate of adverse clinical outcomes compared with patients carrying VUS that were outside of this motif. ${ }^{30}$ These studies together suggest that VUS in sarcomere genes are primarily an admixture of pathogenic and benign variants. So, while patients with HCM carrying sarcomere gene VUS as a whole exhibit a prevalence of clinical outcomes that are intermediate between patients with or without pathogenic sarcomere variants, ${ }^{8}$ a computational approach specifically leveraging the pathogenic mechanism of MYBPC3 has enabled the identification of higher risk subpopulation that exhibit clinical outcomes similar to sarcomeric HCM and a lower risk subpopulation that exhibit clinical outcomes similar to nonsarcomeric HCM.

While computational prediction should not be exclusively relied on to assign pathogenicity of a variant or risk stratify an individual patient, STRUM could be incorporated in an additive manner with other methods for variant adjudication to prioritize variants that warrant further investigation. Given that novel MYBPC3 variants are frequently identified by genetic testing of probands with $\mathrm{HCM}^{29}$ we completed an in silico "saturation mutagenesis" of MYBPC3 compiling a complete list of STRUM+ variants. Excluding known pathogenic or benign variants, we estimate that $\sim 0.097 \%$ $(1 / 1,033)$ individuals within gnomAD carry a MYBPC3 variant predicted to cause subdomain misfolding by STRUM. STRUM+ $M Y B P C 3$ VUS identified in patients with HCM should be prioritized for additional clinical and experimental investigation. Specifically, functional experimental studies to evaluate the direct effects of MYBPC3 VUS on protein stability, folding, and localization, as we have done previously for a subset of pathogenic variants, ${ }^{14}$ will be important. Familial cosegregation analysis on patients carrying a $M Y B P C 3$ STRUM + VUS would add complementary information to these types of experimental studies. 
When known benign missense variants were evaluated by STRUM, 102 of 110 variants were correctly predicted, with an overall specificity of $93 \%$. However, for known pathogenic variants, only 7 of 19 were predicted to alter subdomain folding by STRUM, yielding a sensitivity of $32 \%$. This lower sensitivity was in large part explained by a known cluster of pathogenic variants within $\mathrm{C} 3 .^{15}$ None of the seven known pathogenic variants in $\mathrm{C} 3$ had a $\Delta \Delta G$ value below the threshold of $-1.2 \mathrm{kcal} / \mathrm{mol}$. This is consistent with experimental data that demonstrate C3 variants localize normally to the sarcomere and exhibit protein half lives similar to wild-type MyBP-C. Additionally, an NMR structure of Arg502Trp demonstrates that this variant does not disrupt subdomain folding but rather is more likely to alter protein-protein interactions. ${ }^{14,28}$ In contrast, MyBP-C pathogenic variants in C10, predicted by STRUM to cause subdomain misfolding, fail to localize to the sarcomere and are rapidly degraded. ${ }^{14}$ These experimental results support the accuracy of STRUM predictions for subdomain misfolding. Further, they highlight that STRUM is only predictive of pathogenicity for variants that significantly alter protein folding as their primary mechanism. Thus, a $\Delta \Delta G$ value of $>-1.2 \mathrm{kcal} / \mathrm{mol}$ does not exclude pathogenicity for variants that cause loss or gain-offunction through an alternate mechanism such as alternative splicing or altered protein-protein interactions. STRUM is best applied to VUS after other clinical, computational, and experimental criteria for variant adjudication have been implemented. For example, MYBPC3 pathogenic variants that lead to LoF by mechanisms other than subdomain misfolding have previously been well characterized and defined as pathogenic, including splice variants ${ }^{14,22,23}$ and the cluster of pathogenic variants within C3 (aa.485-503) ${ }^{15,28,31}$ discussed above.

STRUM performed superiorly to sequence based algorithms alone, such as SIFT and PolyPhen-2, which each had lower specificity and were unable to clinically risk stratify patients with $\mathrm{HCM}$ and a MYBPC3 missense VUS. Compared with using each method independently, combining STRUM and CardioBoost improved sensitivity for identifying known pathogenic variants to $63 \%$ while maintaining a specificity for known benign variants of $93 \%$. CardioBoost supported pathogenicity for four missense VUS that were STRUM - but only predicted pathogenicity for 12/34 of MYBPC3 STRUM+ VUS. This result highlights the added utility of STRUM to identify a subset of VUS within MYBPC3 that result in local subdomain misfolding leading to allelic LoF and have a high probability of being pathogenic. Because CardioBoost and STRUM are complementary and have high specificity, we would propose that the ACMG/AMP PP3 criteria, where multiple lines of computational evidence support a deleterious effect of a variant, could be applied when one or both algorithms predict pathogenicity. Conversely, because of relatively limited sensitivity for each algorithm independently, we would propose that the BP4 criteria, where multiple lines of computational evidence support no impact of the variant, be applied only if both algorithms predict that a variant is nonpathogenic.

Although this study was limited by a moderate sample size of 105 patients with HCM, the comprehensive variant adjudication in SHaRe enabled strict inclusion of patients carrying a single VUS within MYBPC3 to clearly discriminate genetic-clinical correlates in this population. This approach enabled us to discern a difference in a composite of adverse clinical outcomes between patients with STRUM+ and STRUM- variants. However, the sample size was insufficient for detecting differences in individual outcomes, such as arrhythmias or heart failure, and did not provide sufficient power to correct for other risk predictors.

The approach of using STRUM as an adjunctive tool for decision making may also be applicable to other genes for which LoF is a pathogenic mechanism. Approximately 50\% of disease associated variants within Human Gene Mutation Database are truncating variants predicted to result in LoF. ${ }^{11}$ These genes, like MYBPC3, also have missense VUS that may be evaluated for protein misfolding using STRUM. For example, there are several causal genes for hypertrophic, dilated, and arrhythmogenic cardiomyopathies with truncating pathogenic variants, including lamin $A / C$, desmoplakin, and plakophilin 2, Titin, and phospholamban. ${ }^{11,32}$ This approach is best suited for nonenzymatic proteins where high-quality structural modeling can be performed, and for which the primary pathogenic mechanism has been established to be LoF.

\section{Conclusions}

We show that the computational algorithm STRUM, that predicts protein structure stability in response to missense variation, enables identification of patients carrying a MYBPC3 VUS who may be at higher clinical risk of adverse events. This approach also provides supportive evidence for pathogenicity, prioritizing variants for functional experimental studies and clinical familial segregation to improve MYBPC3 variant adjudication. Finally, STRUM may be broadly applicable to variants in other genes for which LoF is an established mechanism.

\section{DATA AVAILABILITY}

De-identified data will be made available by request to the authors.

Received: 4 December 2020; Revised: 15 February 2021; Accepted: 16 February 2021;

Published online: 29 March 2021

\section{REFERENCES}

1. Yi, S., Lin, S., Li, Y., Zhao, W., Mills, G. B. \& Sahni, N. Functional variomics and network perturbation: connecting genotype to phenotype in cancer. Nat. Rev. Genet. 18, 395-410 (2017).

2. Starita, L. M. et al. Variant interpretation: functional assays to the rescue. Am. J. Hum. Genet. 101, 315-325 (2017).

3. Richards, S. et al. Standards and guidelines for the interpretation of sequence variants: a joint consensus recommendation of the American College of Medical Genetics and Genomics and the Association for Molecular Pathology. Genet. Med. 17, 405-424 (2015).

4. Balasubramanian, S. et al. Using ALoFT to determine the impact of putative lossof-function variants in protein-coding genes. Nat. Commun. 8, 382 (2017).

5. MacArthur, D. G. et al. A systematic survey of loss-of-function variants in human protein-coding genes. Science. 335, 823-828 (2012).

6. Zhang, X. et al. Disease-specific variant pathogenicity prediction significantly improves variant interpretation in inherited cardiac conditions. Genet. Med. 23, 69-79 (2021).

7. Whiffin, N. et al. CardioClassifier: disease- and gene-specific computational decision support for clinical genome interpretation. Genet. Med. 20, 1246-1254 (2018).

8. Ho, C. Y. et al. Genotype and lifetime burden of disease in hypertrophic cardiomyopathy: insights from the Sarcomeric Human Cardiomyopathy Registry (SHaRe). Circulation. 138, 1387-1398 (2018).

9. Carrier, L., Mearini, G., Stathopoulou, K. \& Cuello, F. Cardiac myosin-binding protein C (MYBPC3) in cardiac pathophysiology. Gene. 573, 188-197 (2015).

10. Marston, $\mathrm{S}$. et al. Evidence from human myectomy samples that MYBPC3 mutations cause hypertrophic cardiomyopathy through haploinsufficiency. Circ. Res. 105, 219-222 (2009).

11. Glazier, A. A., Thompson, A. \& Day, S. M. Allelic imbalance and haploinsufficiency in MYBPC3-linked hypertrophic cardiomyopathy. Pflugers Arch. 471, 781-793 (2019).

12. O'Leary, T. S., Snyder, J., Sadayappan, S., Day, S. M. \& Previs, M. J. MYBPC3 truncation mutations enhance actomyosin contractile mechanics in human hypertrophic cardiomyopathy. J. Mol. Cell. Cardiol. 127, 165-173 (2019).

13. Monteiro da Rocha, A. et al. Deficient CMyBP-C protein expression during cardiomyocyte differentiation underlies human hypertrophic cardiomyopathy cellular phenotypes in disease specific human ES cell derived cardiomyocytes. J. Mol. Cell. Cardiol. 99, 197-206 (2016).

14. Helms, A. S. et al. Spatial and functional distribution of MYBPC3 pathogenic variants and clinical outcomes in patients with hypertrophic cardiomyopathy. Circ. Genom. Precis. Med. 13, 396-405 (2020). 
15. Walsh, R. et al. Quantitative approaches to variant classification increase the yield and precision of genetic testing in Mendelian diseases: the case of hypertrophic cardiomyopathy. Genome Med. 11, 5 (2019).

16. Gersh, B. J. et al. 2011 ACCF/AHA Guideline for the Diagnosis and Treatment of Hypertrophic Cardiomyopathy: a report of the American College of Cardiology Foundation/American Heart Association Task Force on Practice Guidelines. Developed in collaboration with the American Association for Thoracic Surgery, American Society of Echocardiography, American Society of Nuclear Cardiology, Heart Failure Society of America, Heart Rhythm Society, Society for Cardiovascular Angiography and Interventions, and Society of Thoracic Surgeons. J. Am. Coll. Cardiol. 58, e212-260 (2011).

17. Kelly, M. A. et al. Adaptation and validation of the ACMG/AMP variant classification framework for MYH7-associated inherited cardiomyopathies: recommendations by ClinGen's Inherited Cardiomyopathy Expert Panel. Genet. Med. 20, 351-359 (2018).

18. Ko, C. et al. Genetic testing impacts the utility of prospective familial screening in hypertrophic cardiomyopathy through identification of a nonfamilial subgroup. Genet. Med. 20, 69-75 (2018)

19. Quan, L., Lv, Q. \& Zhang, Y. STRUM: structure-based prediction of protein stability changes upon single-point mutation. Bioinformatics. 32, 2936-2946 (2016).

20. Kurolap, A. et al. Establishing the role of PLVAP in protein-losing enteropathy: a homozygous missense variant leads to an attenuated phenotype. J. Med. Genet 55, 779-784 (2018)

21. Amir, M. et al. Impact of Gln94Glu mutation on the structure and function of protection of telomere 1, a cause of cutaneous familial melanoma. J. Biomol. Struct. Dyn. 38, 1514-1524 (2020).

22. Ito, K. et al. Identification of pathogenic gene mutations in LMNA and MYBPC3 that alter RNA splicing. Proc. Natl. Acad. Sci. U. S. A. 114, 7689-7694 (2017).

23. Singer, E. S., Ingles, J., Semsarian, C. \& Bagnall, R. D. Key value of RNA analysis of MYBPC3 splice-site variants in hypertrophic cardiomyopathy. Circ. Genom. Precis. Med. 12, e002368 (2019).

24. Sim, N. L., Kumar, P., Hu, J., Henikoff, S., Schneider, G. \& Ng, P. C. SIFT web server: predicting effects of amino acid substitutions on proteins. Nucleic Acids Res. 40, W452-457 (2012)

25. Kumar, P., Henikoff, S. \& Ng, P. C. Predicting the effects of coding nonsynonymous variants on protein function using the SIFT algorithm. Nat. Protoc. 4, 1073-1081 (2009).

26. Adzhubei, I., Jordan, D. M., Sunyaev, S. R. Predicting functional effect of human missense mutations using PolyPhen-2. Curr. Protoc. Hum. Genet. Chapter 7:Unit7, 20 (2013).

27. Van Driest, S. L. et al. Myosin binding protein C mutations and compound het erozygosity in hypertrophic cardiomyopathy. J. Am. Coll. Cardiol. 44, 1903-1910 (2004).

28. Zhang, X. L., De, S., McIntosh, L. P. \& Paetzel, M. Structural characterization of the C3 domain of cardiac myosin binding protein $C$ and its hypertrophic cardiomyopathy-related R502W mutant. Biochemistry. 53, 5332-5342 (2014).

29. Alfares, A. A. et al. Results of clinical genetic testing of 2,912 probands with hypertrophic cardiomyopathy: expanded panels offer limited additional sensitivity. Genet. Med. 17, 880-888 (2015).

30. Toepfer, C. N. et al. Myosin sequestration regulates sarcomere function, cardiomyocyte energetics, and metabolism, informing the pathogenesis of hypertrophic cardiomyopathy. Circulation. 141, 828-842 (2020).

31. Cohn, R. et al. A contraction stress model of hypertrophic cardiomyopathy due to sarcomere mutations. Stem Cell Rep. 12, 71-83 (2019).

32. Ho, C. Y., Charron, P., Richard, P., Girolami, F., Van Spaendonck-Zwarts, K. Y. \& Pinto, Y. Genetic advances in sarcomeric cardiomyopathies: state of the art. Cardiovasc. Res. 105, 397-408 (2015).

33. Yang, J., Yan, R., Roy, A., Xu, D., Poisson, J. \& Zhang, Y. The I-TASSER Suite: protein structure and function prediction. Nat. Methods 12, 7-8 (2015).

34. Yang, J. \& Zhang, Y. I-TASSER server: new development for protein structure and function predictions. Nucleic Acids Res. 43, W174-181 (2015).

35. Roy, A., Kucukural, A. \& Zhang, Y. I-TASSER: a unified platform for automated protein structure and function prediction. Nat. Protoc. 5, 725-738 (2010).

\section{ACKNOWLEDGEMENTS}

Funding for SHaRe has been provided by an unrestricted research grant by Myokardia, Inc., a startup company that is developing therapeutics that target the sarcomere. Myokardia, Inc had no role in the preparation of this paper or approving the content of this paper. The following individuals are supported by the National Heart, Lung, and Blood Institute (NHLBI) at the National Institutes of Health (NIH): A.D.T. [T32 HL007853], A.S.H [K08HL130455], S.M.D. [R01 11572784], C.Y.H. [1P50HL112349] \& [1U01HL117006]. A.D.T. is supported by the Protein Folding Diseases Initiative (PFDI) and M-BoCA at the
University of Michigan. J.S.W. is supported by the Wellcome Trust [107469/Z/15/Z], the Medical Research Council (United Kingdom), the British Heart Foundation, National Institute for Health Research (NIHR), Royal Brompton Cardiovascular Biomedical Research Unit, and the NIHR Imperial College Biomedical Research Centre. J.I. is a recipient of a National Health and Medical Research Council (NHMRC). C.S. is the recipient of a NHMRC Practitioner Fellowship [1154992]. 1.O. is supported by the Italian Ministry of Health [RF-2013-02356787] and [NET-2011-02347173] and by the Tuscany Registry of Sudden Cardiac Death (ToRSADE) project [FAS-Salute 2014, Regione Toscana]

\section{AUTHOR CONTRIBUTIONS}

Conceptualization: A.D.T., S.M.D. Data curation: A.S.H., N.K.L., S.G.W., A.C.P., D.L.J., S.D.C., E.A.A., S.S., J.S.W., J.I., C.S., M.M., F.M., I.O., C.Y.H. Formal analysis, investigation, methodology: A.D.T., A.K., J.Y., A.S.H. Writing-original draft: A.D.T., S.M.D.; Writingreview \& editing: A.S.H.; N.K.L., S.G.W., A.C.P., D.L.J., S.D.C., E.A.A., S.S., J.S.W., J.I., C.S., M.M., F.M., I.O., C.Y.H.

\section{ETHICS DECLARATION}

Exported data from SHaRe was de-identified. This study complies with the Declaration of Helsinki, Institutional review board and ethics approval was obtained in accordance with policies applicable to each SHaRe site and informed consent was obtained from all participants as required. ShaRe sites are; Brigham and Women's Hospital (Boston, MA, USA), Boston Children's Hospital (Boston, MA, USA), Careggi University (Florence, Italy), Centenary Institute (Sydney, Australia), Children's Hospital of Philadelphia (Philadelphia, PA, USA), Cincinnati Children's Hospital (Cincinnati, OH, USA), Erasmus University (Rotterdam, Netherlands), Laboratory of Genetics and Molecular Cardiology (Sao Paolo, Brazil), Royal Brompton Hospital (London, UK), Royal Prince Alfred Hospital (Sydney, Australia), Stanford University (Palo Alto, CA, USA), University of Michigan (Ann Arbor, MI, USA), University of Pennsylvania (Philadelphia, PA, USA), University of Sydney (Sydney, Australia), Yale-New Haven Hospital (New Haven, CT, USA), Akureyri Hospital Iceland (Akrueyri, Iceland).

\section{COMPETING INTERESTS}

Funding for SHaRe has been provided by an unrestricted research grant by Myokardia, Inc a startup company that is developing therapeutics that target the sarcomere Myokardia, Inc had no role in the preparation of this paper or approving the content of this paper. A.S.H., C.Y.H., S.M.D., S.S., I.O., S.D.C., J.I., and E.A.A. receive research support from MyoKardia, Inc. A.D.T. receives compensation as an editor for Merck Manuals Research funding for all authors is detailed within the Acknowledgements sections of this paper. The other authors declare no competing interests.

\section{ADDITIONAL INFORMATION}

Supplementary information The online version contains supplementary material available at https://doi.org/10.1038/s41436-021-01134-9.

Correspondence and requests for materials should be addressed to A.D.T. or S.M.D.

Reprints and permission information is available at http://www.nature.com/ reprints

Publisher's note Springer Nature remains neutral with regard to jurisdictional claims in published maps and institutional affiliations.

Open Access This article is licensed under a Creative Commons Attribution 4.0 International License, which permits use, sharing, adaptation, distribution and reproduction in any medium or format, as long as you give appropriate credit to the original author(s) and the source, provide a link to the Creative Commons license, and indicate if changes were made. The images or other third party material in this article are included in the article's Creative Commons license, unless indicated otherwise in a credit line to the material. If material is not included in the article's Creative Commons license and your intended use is not permitted by statutory regulation or exceeds the permitted use, you will need to obtain permission directly from the copyright holder. To view a copy of this license, visit http://creativecommons. org/licenses/by/4.0/.

(c) The Author(s) 2021 